\title{
Taxonomic recognition of Saussurea maximowiczii var. triceps on Jejudo Island
}

\author{
Byeol-Ah KIM, Eun-Mi SUN, Seon-Ah YUN ${ }^{1}$, Seung-Cheol KIM ${ }^{1}$ and Hyoung-Tak IM*
}

\author{
Department of Biology, Chonnam National University, Gwangiu 61186, Korea \\ ${ }^{1}$ Department of Biological Sciences, Sungkyunkwan University, Suwon 16419, Korea \\ (Received 8 November 2017; Revised 28 December 2017; Accepted 28 December 2017)
}

\begin{abstract}
A morphological and molecular survey was conducted to determine the taxonomic status of Saussurea maximowiczii var. triceps on Jejudo Island. Although it tends to be smaller than S. maximowiczii with regard to typical quantitative characters of vegetative organs, including the plant height and leaf size, these two taxa cannot be distinguished from each other based on major reproductive characters, including the involucre and florets. Molecular data support the contention that $S$. triceps and S. maximowiczii are monophyletic taxa. Saussurea maximowiczii var. triceps is found to be an ecological phenotype of S. maximowiczii that has adapted particularly to the windy highlands of Hallasan Mountain on Jejudo Island.
\end{abstract}

Keywords: Saussurea triceps, ecophene, highland, Jejudo Island

It was not until the middle of the 19th century that Korean plants became known to the Western plant taxonomists. At that time, plant specimens collected by A. Schlippenbach (1854, East Coast), R. Oldham (1863, South Coast), Y. Hanabusaya (1883-1884), A. W. Carles (1885, Seoul, Incheon, Busan), and R. P. U. Faurie (1900-1907, nationwide) were sent to Leningrad in Russia, KEW Gardens in London, and the University of Tokyo where they were used in various studies. In particular, French Father Emile Joseph Taquet (1873-1952) collected large amount of plant specimens in Hallasan Mountain of Jejudo Island from 1907 to 1910 and sent them to France. Thereafter, many of his specimens were studied by two contemporary French taxonomists, H. Léveille and E. Vaniot.

Based on Taquet's specimens (Taquet 1017, 7 Aug 1908, Yengsil Hallaisan Corea) collected in Yeongsil, Hallasan Mountain, Saussurea triceps H. Lév. \& Vaniot was described as a new species of Saussurea. The former is short in height $(15-25 \mathrm{~cm})$ with hairy involucre and many bracteal leaves at the upper stem. Since then, S. Kitamura of Kyoto University who studied Asteraceae in Northeast Asia treated it as a variety of S. maximowiczii Herd. (see Kitamura, 1937). In a monographic study of Saussurea, S. Lipschitz treated S. triceps as a synonym of $S$. maximowiczii (see Lipschitz, 1979). Since then, taxonomic status of $S$. triceps has been controversial among researchers and literatures, treating it as a synonym of S. maximowiczii (Chen \& Shin, 1999) or a variety of $S$. maximowiczii (Lee, 1996; Im and Lee, 1999). In high elevation of Hallasan Mountain, there are many plants with dwarf forms that have adapted to high mountain winds and low temperatures. $S$. triceps is mainly featured by a dwarf plant that is assumed to be an ecophene. Thus, taxonomic identity of $S$. triceps needs to be confirmed based on plants collected from the type locality.

\section{Materials and Methods}

\section{Morphological study}

To identify geographical distribution of $S$. maximowiczii and S. triceps, we investigated specimens deposited in herbaria of National Institute of Biological Resources (KB), Korea National Arboretum (KH), Chonnam National University (CNU), and Jeju National University (JNUB). To determine the range of intra- and inter- population variations of $S$. maximowiczii and $S$. triceps, we collected specimens of $S$.

\footnotetext{
*Author for correspondence: ihtplant@hanmail.net
} 
maximowiczii from Seonwangsan Mountain population (elev. $200 \mathrm{~m}, 11$ individuals) at Doam-myeon, Hwasun-gun, Jeollanam-do and specimens of $S$. triceps from 1,100-m altitude highland population (elev. 1,100 m, 6 individuals) at Seogwipsi, Jejudo Island. A total of 53 individuals derived from Hwasun, Seonwangsan Mountain population and Jeju 1,100$\mathrm{m}$ highland population, CNU (15 collections) and JNUB (21 collections) were examined for their morphological traits such as stem length, leaves, involucre, and florets to determine the range of morphological variations of $S$. maximowiczii and $S$. triceps (Table 1). In Eorimok (high elevation of Hallasan Mountain), 1,100-m altitude wetlands, and Yeongsil, we conducted on-site study regarding ecophene plants.

\section{Molecular study}

\section{Plant samples}

In the present study, 18 species and 29 taxa of Saussurea distributed across the Korean peninsula were used (Table 2). All plant samples endemic to Korea were collected from July 2013 to August 2017. We collected S. maximowiczii from Biseulsan Mountain (Daegu), Mudeungsan Mountain (Gwangju), Baegunsan Mountain (Chonnam), and Chilbosan Mountain (Gyeonggi-do). We also collected S. triceps from Hallasan Mountain (Jeju's 1,100-m altitude highland). For $S$. myokoensis, its field collection was difficult. Therefore, specimens deposited in KH were used. Carduus crispus (GenBank accession EF010530) was used as an outgroup (Table 2).

Table 1. Examined specimen and each morphological character.

\begin{tabular}{|c|c|c|c|c|c|c|c|c|c|c|c|}
\hline \multirow{2}{*}{$\begin{array}{l}\text { Specimen } \\
\text { Number }\end{array}$} & \multirow[b]{2}{*}{ Locality } & \multicolumn{2}{|c|}{ Stem } & \multicolumn{2}{|c|}{ Leaf blade } & \multicolumn{2}{|c|}{ Involucre } & \multicolumn{2}{|c|}{ Floret } & \multirow{2}{*}{$\begin{array}{c}\text { Achene } \\
\text { (mm) }\end{array}$} & \multirow{2}{*}{$\begin{array}{c}\text { Pappus } \\
(\mathrm{mm})\end{array}$} \\
\hline & & $\begin{array}{l}\text { Width } \\
(\mathrm{mm})\end{array}$ & $\begin{array}{l}\text { Height } \\
(\mathrm{cm})\end{array}$ & $\begin{array}{l}\text { Length } \\
\text { (cm) }\end{array}$ & $\begin{array}{l}\text { Width } \\
\text { (cm) }\end{array}$ & $\begin{array}{l}\text { Length } \\
\text { (cm) }\end{array}$ & $\begin{array}{l}\text { Width } \\
\text { (cm) }\end{array}$ & $\begin{array}{l}\mathrm{NP} \\
(\mathrm{mm})\end{array}$ & $\begin{array}{l}\text { WP } \\
(\mathrm{mm})\end{array}$ & & \\
\hline $\begin{array}{l}\max \\
\mathrm{CNU} 222071\end{array}$ & $\begin{array}{l}\text { JN, Jangseong, } \\
\text { Mt. Ibamsan }\end{array}$ & 5.8 & 136 & 39 & 12 & 97 & 5.6 & 6.7 & 4.5 & 6.2 & 8.2 \\
\hline $\begin{array}{l}\max \\
\mathrm{CNU} 222072\end{array}$ & $\begin{array}{l}\text { JN, Jangseong, } \\
\text { Mt. Ibamsan }\end{array}$ & 5.1 & 125 & 23 & 8.2 & 106 & 5.9 & 6 & 4.3 & 5.3 & 8 \\
\hline $\begin{array}{l}\max \\
\mathrm{CNU} 222073\end{array}$ & $\begin{array}{l}\text { JN, Jangseong, } \\
\text { Mt. Ibamsan }\end{array}$ & 6.7 & 138 & 32 & 10 & 89 & 5.4 & 6.9 & 4.6 & 5.5 & 7.2 \\
\hline $\begin{array}{l}\max \\
\mathrm{CNU} 222074\end{array}$ & $\begin{array}{l}\text { JN, Jangseong, } \\
\text { Mt. Ibamsan }\end{array}$ & 5.2 & 110 & 31 & 9.8 & 113 & 6.2 & 6.9 & 4.5 & 5.2 & 8.6 \\
\hline $\begin{array}{l}\max \\
\mathrm{CNU} 010017\end{array}$ & $\begin{array}{l}\text { JN, Suncheon, } \\
\text { Mt. Jogyesan }\end{array}$ & 4.1 & 98 & 21 & 6.7 & 112 & 5.9 & 5.5 & 3 & 5.4 & 8.2 \\
\hline $\begin{array}{l}\max \\
\text { CNU } 384686\end{array}$ & $\begin{array}{l}\text { JN, Sinan, } \\
\text { Josamji }\end{array}$ & 2.9 & 112 & 14 & 4.2 & 97 & 5.8 & 5.6 & 3.1 & 5.4 & 8.6 \\
\hline $\begin{array}{l}\max \\
\text { CNU } 059303\end{array}$ & $\begin{array}{l}\text { JN, Sinan, } \\
\text { Isl. Aphaedo }\end{array}$ & 2.7 & 80 & 16 & 5.5 & 108 & 6 & 5.6 & 3.2 & 6.3 & 8.6 \\
\hline $\begin{array}{l}\max \\
\mathrm{CNU} 010018\end{array}$ & $\begin{array}{l}\text { JN, Suncheon, } \\
\text { Mt. Jogyesan }\end{array}$ & 4.2 & 65 & 15 & 4.4 & 121 & 5.7 & 6.8 & 3.6 & 6 & 8.2 \\
\hline $\begin{array}{l}\max \\
\mathrm{CNU} 074404\end{array}$ & GG, Hwasong & 5.4 & 117 & 31 & 10.2 & 107 & 5.7 & 5.7 & 3.5 & 6.1 & 7.5 \\
\hline $\begin{array}{l}\max \\
\text { CNU } 040247\end{array}$ & $\begin{array}{l}\text { JN, Haenam, } \\
\text { Dusolbong }\end{array}$ & 3.5 & 86 & 19 & 6.5 & 124 & 5.8 & 5.6 & 3.5 & 5.3 & 8.3 \\
\hline $\begin{array}{l}\max \\
\text { CNU } 040248\end{array}$ & $\begin{array}{l}\text { JN, Sinan, } \\
\text { Isl. Imjado }\end{array}$ & 2.7 & 32 & 8 & 3.2 & 104 & 5.6 & 6.1 & 3.6 & 5.6 & 7.3 \\
\hline $\begin{array}{l}\max \\
\text { CNU } 057246\end{array}$ & $\begin{array}{l}\text { JN, Haenam, } \\
\text { Mt. } \\
\text { Geumgangsan }\end{array}$ & 6 & 127 & 27 & 9.1 & 121 & 5.7 & 6.3 & 3.7 & 5.5 & 7.7 \\
\hline $\begin{array}{l}\max \\
\text { CNU } 050988\end{array}$ & $\begin{array}{l}\text { JN, Gangjin, } \\
\text { Mt. Jujaksan }\end{array}$ & 2.8 & 117 & 16 & 5.6 & 121 & 6.3 & 5.5 & 3 & 5.9 & 8.5 \\
\hline $\begin{array}{l}\max \\
\text { CNU } 099394\end{array}$ & $\begin{array}{l}\text { JB, Sunchang, } \\
\text { Mt. Chuwolsan }\end{array}$ & 3 & 94 & 14 & 4.5 & 119 & 6.1 & 6.2 & 3.4 & 5.8 & 8.4 \\
\hline $\begin{array}{l}\max \\
\text { CNU } 013190\end{array}$ & $\begin{array}{l}\text { JN, Sinan, } \\
\text { Mt. Hwahaksan }\end{array}$ & 2.7 & 45 & 7 & 3.3 & 112 & 4.9 & 5.7 & 3.3 & 5.7 & 7.9 \\
\hline
\end{tabular}


Table 1. Continued.

\begin{tabular}{|c|c|c|c|c|c|c|c|c|c|c|c|}
\hline \multirow{2}{*}{$\begin{array}{c}\text { Specimen } \\
\text { Number }\end{array}$} & \multirow[b]{2}{*}{ Locality } & \multicolumn{2}{|c|}{ Stem } & \multicolumn{2}{|c|}{ Leaf blade } & \multicolumn{2}{|c|}{ Involucre } & \multicolumn{2}{|c|}{ Floret } & \multirow{2}{*}{$\begin{array}{l}\text { Achene } \\
(\mathrm{mm})\end{array}$} & \multirow{2}{*}{$\begin{array}{c}\text { Pappus } \\
(\mathrm{mm})\end{array}$} \\
\hline & & $\begin{array}{l}\text { Width } \\
(\mathrm{mm})\end{array}$ & $\begin{array}{l}\text { Height } \\
(\mathrm{cm})\end{array}$ & $\begin{array}{l}\text { Length } \\
(\mathrm{cm})\end{array}$ & $\begin{array}{l}\text { Width } \\
(\mathrm{cm})\end{array}$ & $\begin{array}{l}\text { Length } \\
(\mathrm{cm})\end{array}$ & $\begin{array}{l}\text { Width } \\
(\mathrm{cm})\end{array}$ & $\begin{array}{c}\mathrm{NP} \\
(\mathrm{mm})\end{array}$ & $\begin{array}{l}\text { WP } \\
(\mathrm{mm})\end{array}$ & & \\
\hline $\max$ HS 1 & $\begin{array}{l}\text { JN, Hwasun, } \\
\text { Mt. } \\
\text { Seonwangsan }\end{array}$ & 3.4 & 86 & 30 & 13 & 9 & $4 . .7$ & 6.7 & 4.4 & 5.9 & 8.1 \\
\hline $\max$ HS 2 & $\begin{array}{l}\text { JN, Hwasun, } \\
\text { Mt. } \\
\text { Seonwangsan }\end{array}$ & 5.4 & 100 & 22 & 11 & 11.6 & 4.2 & 5.9 & 3.8 & 5.6 & 8 \\
\hline $\max$ HS 3 & $\begin{array}{l}\text { JN, Hwasun, } \\
\text { Mt. } \\
\text { Seonwangsan }\end{array}$ & 5.2 & 97 & 28 & 14 & 12.1 & 4.6 & 6.9 & 4.5 & 6.2 & 8.3 \\
\hline $\max$ HS 4 & $\begin{array}{l}\text { JN, Hwasun, } \\
\text { Mt. } \\
\text { Seonwangsan }\end{array}$ & 3.2 & 80 & 27 & 15 & - & - & - & - & - & - \\
\hline $\max$ HS 5 & $\begin{array}{l}\text { JN, Hwasun, } \\
\text { Mt. } \\
\text { Seonwangsan }\end{array}$ & 3.3 & 82 & 25 & 11 & 10.6 & 4.1 & 6.5 & 4.4 & 6 & 8.2 \\
\hline $\max$ HS 6 & $\begin{array}{l}\text { JN, Hwasun, } \\
\text { Mt. } \\
\text { Seonwangsan }\end{array}$ & 3.5 & 107 & 26 & 11.5 & 11.2 & 3.9 & 6.3 & 4.1 & 5.6 & 7.7 \\
\hline $\max$ HS 7 & $\begin{array}{l}\text { JN, Hwasun, } \\
\text { Mt. } \\
\text { Seonwangsan }\end{array}$ & 3.8 & 67 & 22 & 9 & 12 & 5.2 & 6.5 & 4.3 & 5.8 & 7.6 \\
\hline $\max$ HS 8 & $\begin{array}{l}\text { JN, Hwasun, } \\
\text { Mt. } \\
\text { Seonwangsan }\end{array}$ & 6.4 & 134 & 28 & 16.5 & 11.7 & 4.6 & 5.7 & 3.7 & 6.1 & 8 \\
\hline $\max$ HS 9 & $\begin{array}{l}\text { JN, Hwasun, } \\
\text { Mt. } \\
\text { Seonwangsan }\end{array}$ & 4.1 & 128 & 23 & 13.5 & 10 & 4.1 & 6 & 4 & 6.2 & 8.5 \\
\hline $\max$ HS 10 & $\begin{array}{l}\text { JN, Hwasun, } \\
\text { Mt. } \\
\text { Seonwangsan }\end{array}$ & 3.2 & 60 & 22 & 9.5 & 10.2 & 4.1 & 6.4 & 4.2 & 5.3 & 7.8 \\
\hline $\max$ HS 11 & $\begin{array}{l}\text { JN, Hwasun, } \\
\text { Mt. } \\
\text { Seonwangsan }\end{array}$ & 3.8 & 106 & 29 & 13.5 & 10.5 & 4.2 & - & - & - & - \\
\hline tri 11001 & $\begin{array}{l}\text { JJ, } 1100 \\
\text { Highland }\end{array}$ & 1.4 & 23 & 6 & 2.2 & - & - & - & - & - & - \\
\hline tri 11002 & $\begin{array}{l}\text { JJ, } 1100 \\
\text { Highland }\end{array}$ & 1.5 & 26 & 6 & 1.8 & - & - & - & - & - & - \\
\hline tri 11003 & $\begin{array}{l}\text { JJ, } 1100 \\
\text { Highland }\end{array}$ & 2.3 & 48 & 8 & 3.3 & - & - & - & - & - & - \\
\hline tri 11004 & $\begin{array}{l}\text { JJ, } 1100 \\
\text { Highland }\end{array}$ & 2.4 & 20 & 5 & 1.8 & 11 & 4.6 & 6.9 & 4.5 & 6.3 & 8.4 \\
\hline tri 11005 & $\begin{array}{l}\text { JJ, } 1100 \\
\text { Highland }\end{array}$ & 2.1 & 22 & 5 & 1.7 & - & - & - & - & - & - \\
\hline tri 11006 & $\begin{array}{l}\text { JJ, } 1100 \\
\text { Highland }\end{array}$ & 2 & 15 & 7 & 2.6 & - & - & - & - & - & - \\
\hline
\end{tabular}


Table 1. Continued.

\begin{tabular}{|c|c|c|c|c|c|c|c|c|c|c|c|}
\hline \multirow{2}{*}{$\begin{array}{l}\text { Specimen } \\
\text { Number }\end{array}$} & \multirow[b]{2}{*}{ Locality } & \multicolumn{2}{|c|}{ Stem } & \multicolumn{2}{|c|}{ Leaf blade } & \multicolumn{2}{|c|}{ Involucre } & \multicolumn{2}{|c|}{ Floret } & \multirow{2}{*}{$\begin{array}{l}\text { Achene } \\
(\mathrm{mm})\end{array}$} & \multirow{2}{*}{$\begin{array}{c}\text { Pappus } \\
(\mathrm{mm})\end{array}$} \\
\hline & & $\begin{array}{l}\text { Width } \\
(\mathrm{mm})\end{array}$ & $\begin{array}{l}\text { Height } \\
(\mathrm{cm})\end{array}$ & $\begin{array}{l}\text { Length } \\
(\mathrm{cm})\end{array}$ & $\begin{array}{l}\text { Width } \\
(\mathrm{cm})\end{array}$ & $\begin{array}{l}\text { Length } \\
(\mathrm{cm})\end{array}$ & $\begin{array}{l}\text { Width } \\
(\mathrm{cm})\end{array}$ & $\begin{array}{l}\mathrm{NP} \\
(\mathrm{mm})\end{array}$ & $\begin{array}{c}\text { WP } \\
(\mathrm{mm})\end{array}$ & & \\
\hline $\begin{array}{l}\text { tri } \\
\text { JJU } 009951\end{array}$ & $\begin{array}{l}\text { JJ, } 1100 \\
\text { Highland }\end{array}$ & 1.9 & 9 & 5 & 1.7 & 11.3 & 4.3 & 6.7 & 4.1 & 6 & 8.6 \\
\hline $\begin{array}{l}\text { tri } \\
\text { JJU } 0013993\end{array}$ & $\begin{array}{l}\text { JJ, } 1100 \\
\text { Highland }\end{array}$ & 2.9 & 22 & 5 & 1.8 & 10.7 & 5.6 & 5.7 & 3.6 & 5.9 & 8.1 \\
\hline $\begin{array}{l}\text { tri } \\
\text { JJU } 0013984\end{array}$ & $\begin{array}{l}\text { JJ, } 1100 \\
\text { Highland }\end{array}$ & 2.3 & 36 & 10 & 4 & 10.6 & 5 & 5.9 & 4 & 6.1 & 8.1 \\
\hline $\begin{array}{l}\text { tri } \\
\text { JJU } 010922\end{array}$ & $\begin{array}{l}\text { JJ, } 1100 \\
\text { Highland }\end{array}$ & 2.7 & 30 & 7 & 2.4 & 10 & 4.8 & - & - & - & - \\
\hline $\begin{array}{l}\text { tri } \\
\text { JJU } 0013982\end{array}$ & $\begin{array}{l}\text { JJ, } 1100 \\
\text { Highland }\end{array}$ & 3.1 & 30 & 7 & 2.3 & 10.3 & 4.9 & 6.2 & 4.1 & 5.7 & 7.7 \\
\hline $\begin{array}{l}\text { tri } \\
\text { JJU } 0013999\end{array}$ & $\begin{array}{l}\text { JJ, } 1100 \\
\text { Highland }\end{array}$ & 2.2 & 56 & 10 & 3.7 & 11.4 & 5.1 & 5.5 & 3.3 & 6.1 & 8 \\
\hline $\begin{array}{l}\text { tri } \\
\text { JJU } 0003697\end{array}$ & $\begin{array}{l}\text { JJ, } 1100 \\
\text { Highland }\end{array}$ & 1.4 & 17 & 6 & 2.6 & 10.9 & 4.1 & 6 & 4 & 5.8 & 7.8 \\
\hline $\begin{array}{l}\text { tri } \\
\text { JJU } 011118\end{array}$ & $\begin{array}{l}\text { JJ, } 1100 \\
\text { Highland }\end{array}$ & 1.3 & 22 & 6 & 1.2 & 11.2 & 5.1 & 6.2 & 4.3 & 6.1 & 8.1 \\
\hline $\begin{array}{l}\text { tri } \\
\text { JJU } 0013936\end{array}$ & $\begin{array}{l}\text { JJ, } 1100 \\
\text { Highland }\end{array}$ & 3.9 & 47 & - & - & 11.4 & 6 & - & - & - & - \\
\hline $\begin{array}{l}\text { tri } \\
\text { JJU } 0013997\end{array}$ & $\begin{array}{l}\text { JJ, } 1100 \\
\text { Highland }\end{array}$ & 2.6 & 26 & 8 & 4 & 12 & 6.4 & 6.7 & 4.4 & 5.7 & 7.6 \\
\hline $\begin{array}{l}\text { tri } \\
\text { JJU } 0013988\end{array}$ & $\begin{array}{l}\text { JJ, } 1100 \\
\text { Highland }\end{array}$ & 1.7 & 33 & 7 & 2.6 & 12.2 & 4.8 & 6 & 3.9 & 5.8 & 7.7 \\
\hline $\begin{array}{l}\text { tri } \\
\text { JJU } 0019050\end{array}$ & $\begin{array}{l}\text { JJ, } 1100 \\
\text { Highland }\end{array}$ & 2 & 36 & 7 & 3 & 11.4 & 5.5 & 6.7 & 4.1 & 6.1 & 8 \\
\hline $\begin{array}{l}\text { tri } \\
\text { JJU } 0019064\end{array}$ & $\begin{array}{l}\text { JJ, } 1100 \\
\text { Highland }\end{array}$ & 2.7 & 29 & 9 & 3.5 & 12.4 & 5.9 & 6.9 & 4.2 & 5.9 & 7.9 \\
\hline $\begin{array}{l}\text { tri } \\
\text { JJU } 0013980\end{array}$ & $\begin{array}{l}\text { JJ, } 1100 \\
\text { Highland }\end{array}$ & 2 & 49 & 10 & 4.8 & 10.3 & 4.2 & 6.1 & 4.1 & 6.4 & 8.4 \\
\hline $\begin{array}{l}\text { tri } \\
\text { JJU } 0005776\end{array}$ & $\begin{array}{l}\text { JJ, } 1100 \\
\text { Highland }\end{array}$ & 2.9 & 31 & 9 & 5.1 & 11.4 & 4.6 & 5.9 & 4.1 & 5.5 & 7.3 \\
\hline $\begin{array}{l}\text { tri } \\
\text { JJU } 0013986\end{array}$ & $\begin{array}{l}\text { JJ, Mansudong- } \\
\text { san }\end{array}$ & 2.3 & 32 & 10 & 3.4 & 11.1 & 4.5 & 6.1 & 4.2 & 5.4 & 7.5 \\
\hline $\begin{array}{l}\text { tri } \\
\text { JJU } 0013995\end{array}$ & JJ, Eorimok & 2.6 & 38 & 8 & 3.2 & 9.8 & 4.6 & 5.6 & 3 & 6.2 & 8.1 \\
\hline $\begin{array}{l}\text { tri } \\
\text { JJU } 0001404\end{array}$ & $\mathrm{JJ}$, Eorimok & 2 & 23 & 6 & 2 & 9.8 & 5 & 5.9 & 3.7 & 6 & 8 \\
\hline $\begin{array}{l}\text { tri } \\
\text { JJU } 0001401\end{array}$ & $\begin{array}{l}\text { JJ, summit of Mt. } \\
\text { Hallasan }\end{array}$ & 2.3 & 45 & 12 & 9.2 & 10.3 & 4.3 & 6.5 & 4.1 & 5.5 & 7.2 \\
\hline $\begin{array}{l}\text { tri } \\
\text { JJU } 009947\end{array}$ & JJ, Sangumburi & 3 & 68 & 13 & 4 & 10.7 & 4.2 & 6.2 & 4.3 & 5.7 & 7.8 \\
\hline $\begin{array}{l}\text { tri } \\
\text { JJU } 009946\end{array}$ & JJ, Sangumburi & 2.6 & 58 & 11 & 4.3 & 10.4 & 4.2 & - & - & - & -- \\
\hline
\end{tabular}

NP, narrow part of floral tube; WP, wide part of floral tube; max, S. maximowiczii; tri, S. maximowiczii var. tricps; JN, Jeollanam-do; JB, Jeollabuk-do; JJ, Jeju-do; CNU, Herbarium of Chonnam National University; JJU, Herbarium of Jeju National University; HS, Hwasun population; 1100, Jejudo Isl. 1100-m altitude Highland. 
Table 2. List of samples used in this study.

\begin{tabular}{|c|c|c|}
\hline Abbreviation & Taxa & Locality \\
\hline DD & Saussurea calcicola Nakai & Pyeongchang-gun, Gangwon-do \\
\hline DH & Saussurea chabyoungsanica H. T. Im & Taebaek-si, Gangwon-do \\
\hline SA & Saussurea diamantica Nakai & Yangyang-gun, Gangwon-do \\
\hline $\mathrm{HB}$ & Saussurea eriophylla Nakai & Jeongseon-gun, Gangwon-do \\
\hline HA & Saussurea gracilis Maxim. & Gapyeong-gun, Gyeonggi-do \\
\hline GA & Saussurea gracilis Maxim. & Hapcheon-gun, Gyeongsangnam-do \\
\hline HA & Saussurea grandicapitula W. Lee et H. T. Im & Gapyeong-gun, Gyeonggi-do \\
\hline AS & Saussurea grandifolia Maxim. & Inje-gun, Gangwon-do \\
\hline EL & Saussurea grandifolia Maxim. & Ulleung-gun, Gyeongsangbuk-do \\
\hline GR & Saussurea macrolepis (Nakai) Kitam. & Namwon-si, Jeollabuk-do \\
\hline BS & Saussurea maximowiczii Herder & Dalseong-gun, Daegu \\
\hline MD & Saussurea maximowiczii Herder & Buk-gu, Gwangju \\
\hline BU & Saussurea maximowiczii Herder & Gwangyang-si, Jeollanam-do \\
\hline $\mathrm{CD}$ & Saussurea maximowiczii Herder & Hwaseong-si, Gyeonggi-do \\
\hline $\mathrm{JJ}$ & Saussurea maximowiczii var triceps & Seogwipo-si, Jeju-do \\
\hline $\mathrm{BL}$ & Saussurea mongolica.(Franch.) Franch. & Jeongseon-gun, Gangwon-do \\
\hline DD & Saussurea mongolica (Franch.) Franch & Pyeongchang-gun, Gangwon-do \\
\hline MH & Saussurea myokoensis Kitam. & Korea National Arboretum specimen \\
\hline OR & Saussurea odontolepis Sch. Bip. ex Herder & Yeongwol-gun, Gangwon-do \\
\hline TA & Saussurea odontolepis Sch. Bip. ex Herder & Taean-gun, Chungcheongnam-do \\
\hline HS & Saussurea polylepis Nakai & Sinan-gun, Jeollanam-do \\
\hline HD & Saussurea polylepis Nakai & Sinan-gun, Jeollanam-do \\
\hline GY & Saussurea pulchella (Fisch.) Fisch. ex Colla & Jeongseon-gun, Gangwon-do \\
\hline OD & Saussurea pulchella (Fisch.) Fisch. ex Colla & Hongcheon-gun, Gangwon-do \\
\hline HA & Saussurea seoulensis Nakai & Gapyeong-gun, Gyeonggi-do \\
\hline $\mathrm{KD}$ & Saussurea seoulensis Nakai & Pocheon-si, Gyeonggi-do \\
\hline AS & Saussurea tanakae Franch. \& Sav. ex Maxim. & Inje-gun, Gangwon-do \\
\hline DH & Saussurea tanakae Franch. \& Sav. ex Maxim. & Taebaek-si, Gangwon-do \\
\hline $\mathrm{JJ}$ & Saussurea taquetii H. Lév. \& Vaniot & Seogwipo-si, Jeju-do \\
\hline
\end{tabular}

\section{DNA extraction and PCR}

Both fresh and dried samples (stored in silica gel) of each plant material were collected prior to DNA extraction. Total genomic DNA was isolated from $20-25 \mathrm{mg}$ of dried leaves using DNeasy Plant Mini Kit (Qiagen, Hilden, Germany) according to the manufacturer's protocol. PCR products were electrophoresed on $1 \%$ agarose gel to visualize characteristic band patterns to differentiate between DNA fragments. To investigate molecular variations, PCR utilized internal transcribed spacer (ITS), $t r n \mathrm{~L}-t r n \mathrm{~F}, p s b \mathrm{~A}-\operatorname{tr} n \mathrm{H}$, and $r b c \mathrm{~L}$ based on previous molecular phylogenetic studies of Saussurea
(Raab-Straube et al., 2003; Kita et al., 2004; Wang et al., 2007, 2009, 2013). In addition, we used trnQ-5'rps 16 and psbJ-petA because they had high potentially informative character and percentage variability values (Shaw et al., 2007). Six regions of DNA were amplified: one nuclear DNA region and five chloroplast DNA (cpDNA) regions. PCR for amplification of DNA preparations was carried out with the following components: $1 \mu \mathrm{L}$ of template DNA, $5 \mu \mathrm{L}$ of $10 \times$ Taq polymerase reaction buffer, $1 \mu \mathrm{L}$ each of $10 \mathrm{mM}$ dNTPs mix, $1 \mu \mathrm{L}$ each of 10 pmol primer, $0.2 \mu \mathrm{L}$ of 1.25 units of DiastarTaq DNA polymerase (SolGent Co., Daejeon, Korea), and 
Table 3. Characteristics of ITS and combined cpDNA datasets obtained from maximum parsimony analyses.

\begin{tabular}{lcc}
\hline \hline & ITS & Combined cpDNA \\
\hline $\begin{array}{l}\text { Number of taxa } \\
\text { (including 1 outgroup) }\end{array}$ & 30 & 30 \\
Total length & 647 & $\begin{array}{c}3,346 \\
\text { (trnL-trnF 852, rbcL 503, } \\
\text { psbJ-petA 795, trnQ-5'rps 16 } \\
798, p s b \text { A-trnH 398) }\end{array}$ \\
$\begin{array}{lcc}\text { Number of parsimony } \\
\text { informative sites }\end{array}$ & 68 & 25 \\
Number of parsimony & 57 & 43 \\
$\quad$ uninformative sites & & 3278 \\
Number of constant sites & 522 & 77 \\
Tree length & 161 & 0.896 \\
Consistency index & 0.851 & 0.906 \\
Retention index & 0.919 & \\
\hline
\end{tabular}

ITS, internal transcribed spacer; cpDNA, chloroplast DNA.

distilled water to make a total volume of $50 \mu \mathrm{L}$. PCR cycling conditions were: template denaturation at $95^{\circ} \mathrm{C}$ for $1 \mathrm{~min}$ followed by primer annealing at $54-58^{\circ} \mathrm{C}$ for $1 \mathrm{~min}$ and primer extension at $72^{\circ} \mathrm{C}$ for $2 \mathrm{~min}$. We repeated the above denaturation, annealing, and extension steps with 35-40 cycles.

\section{Sequence alignment and phylogenetic analysis}

Editing and alignment of all sequences were conducted using Geneious version 10.2.2 (Kearse et al., 2012). Gaps were treated as missing characters and all traits were equally weighted. Each data matrix (Table 3) was subjected to maximum parsimony (MP) and maximum likelihood (ML) for taxonomic analysis. ML was analyzed using PAUP* 4.0b10 (Swofford, 2001). MP analyses involved a heuristic search strategy with tree bisection reconnection branch swapping option. ML analyses were conducted using IQ-TREE software (Nguyen et al., 2015). TIM3e+G4 was selected as the fittest model of ITS. Furthermore, F81+I was selected for combined cpDNA data. To investigate statistical support of each cladogram, bootstrap values were calculated from 1,000 replicates (Felsenstein, 1985).

\section{Results and Discussion}

\section{External morphology}

According to protologue and description in the name combination of Saussurea maximowiczii and S. triceps, the difference between the two taxa is that the latter is shorter in height than the former and lower leaves of $S$. triceps are shorter. Morphological traits such as involucral bract, florets, achene, and pappus have been used as important criteria of Saussurea classification in previous studies (Im, 1989, 1990, 1991). For 53 individuals used for morphometry, morphological traits were examined to determine the range of morphological variations of $S$. maximowiczii and S. triceps (Table 1). For some individuals that were not fully mature, there were some deficiencies in the measurement.

For quantitative traits such as height, stem diameter, and lower leaf size, S. triceps from the 1100-m latitude Highland population tended to be smaller than those of $S$. maximowiczii from Seonwangsan Mountain population. However, this variation is continuous between populations and within populations. Such continuity becomes clear when heights of samples collected from the 1100-m altitude Highland population and the Seonwangsan Mountain population are compared (Fig. 1). This is also true in the graphs based on specimens deposited in the herbaria (Fig. 2). The quantitative trait represented by plant height is generally a trait that has wide range of variations because it is highly affected by the environment.

In contrast, reproductive organs such as involucral bract, florets, achene, and pappus are less affected by the environment. For traits of involucral bract, florets, achene, and pappus, S. triceps which consists of 1100-m altitude Highland population and $S$. maximiziczii, including Seonwangsan Mountain population are mixed within narrow variation range (Table 1). Florets of Saussurea consist of tube and limb. The limb is divided into five lobes. The tube is divided into a narrow part at the bottom and a broad part at the top (Fig. 3). Lengths of the narrow part and the broad part are very stable traits (Im, 1989). When lengths of the broad and narrow parts of florets are plotted on a graph, $S$. maximowiczii and $S$. triceps cannot be distinguished (Figs. 4, 5).

\section{Molecular data}

Phylogenetic tree based on ITS nucleotide sequence of nuclear DNA revealed that $S$. triceps and $S$. maximowiczii formed a monophyletic group supported by MP bootstrap (BS) $=100 \%$ and ML BS $=100 \%$ (Fig. 7). This demonstrates that $S$. triceps and $S$. maximowiczii share most recent common ancestor regardless of their taxonomic position or species distinctness. However, $S$. triceps/S. maximowiczii clade is well segregated from other Saussurea taxa distributed on the Korean peninsula.

Phylogenetic tree based on five regions of cpDNA was 
similar to the ITS tree in which $S$. triceps and $S$. maximowiczii formed a clade. However, two clades which were not detected in the ITS tree were observed in the cpDNA tree: Clade 1 consisted of S. maximowiczii from Mudeungsan Mountain and Biseulsan Mountain, with $63 \%$ of MP BS and $92 \%$ of ML BS; Clade 2 consisted of $S$. maximowiczii from Baekunsan
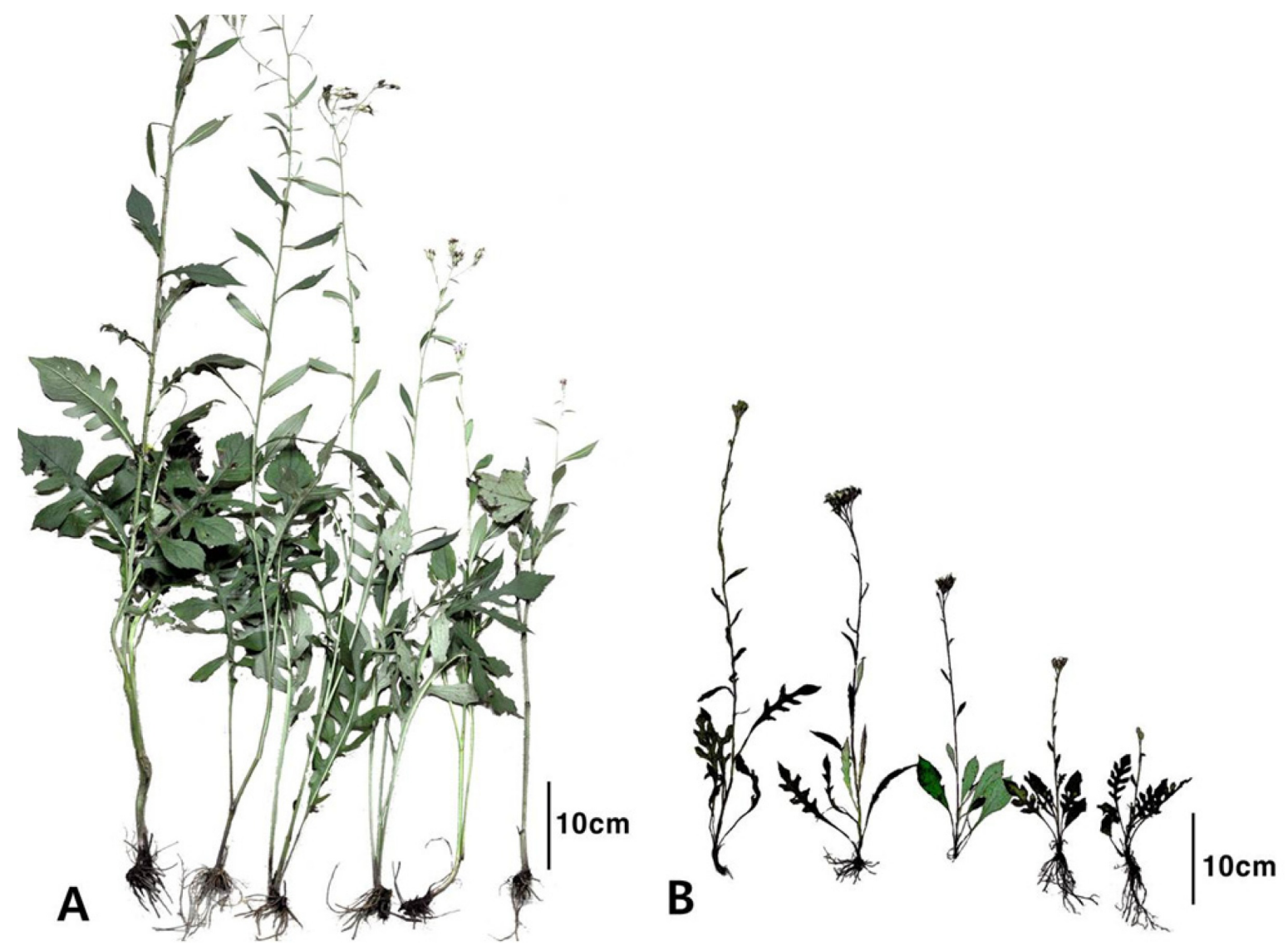

Fig. 1. Plants from Hwasun Seonwangsan population (A; Saussurea maximowiczii) and Jeju 1100-m altitude Highland population (B; S. maximowiczii var. triceps).

\section{Height}

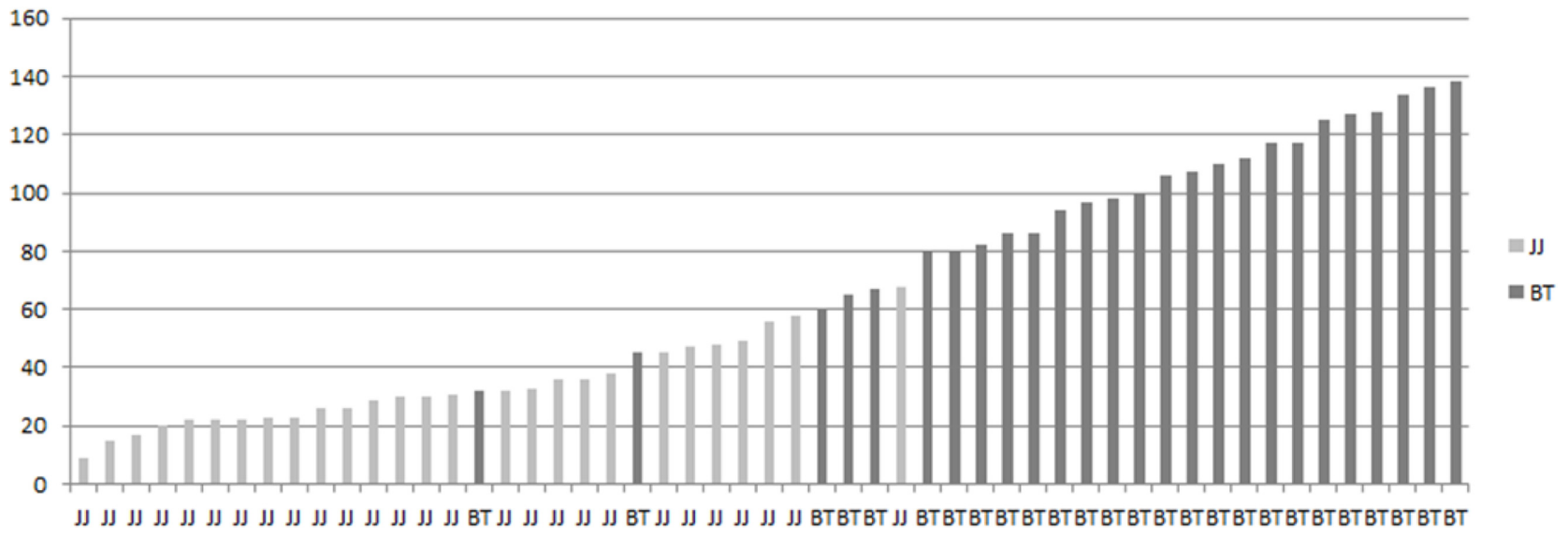

Fig. 2. Plants height of Saussurea maximowiczii (BT) and S. maximowiczii var. triceps (JJ). 


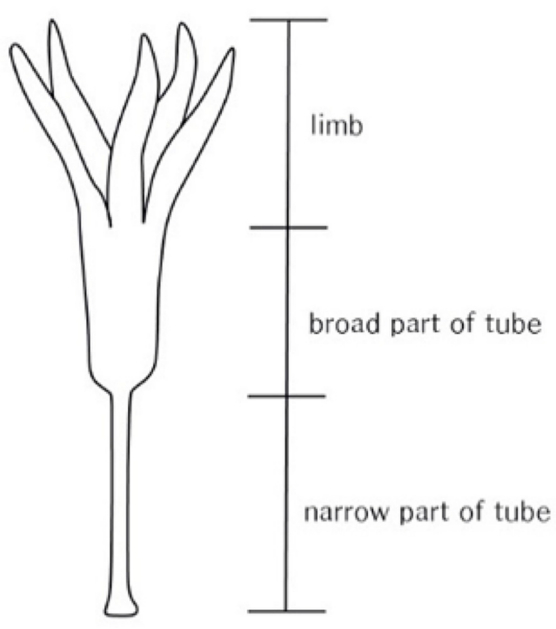

Fig. 3. Floret of genus Saussurea.
Mountain and $S$. triceps from Jejudo Island, with $61 \%$ of MP BS and $93 \%$ of ML BS (Fig. 8). A sister group relationship between $S$. triceps and $S$. maximowiczii of Baekunsan Mountain implies their geographical correlation.

These results support the possibility that $S$. triceps is an ecophene of $S$. maximowiczii. However, it cannot be ruled out that $S$. triceps may maintain a species position. The distribution of $S$. triceps is confined to Jejudo Island which has quite different environmental conditions from the inland area. Populations of $S$. triceps are relatively small in size. Thus, it is easily affected by founder effect, inbreeding, and so that that may lead to wide morphological variations among subpopulations. In addition, it is isolated from $S$. maximowiczii distributed across the Korean peninsula. S. triceps may maintain genetic traits by cutting off genetic interactions with $S$. maximowiczii. $S$.

\section{Length of broad part of tube}

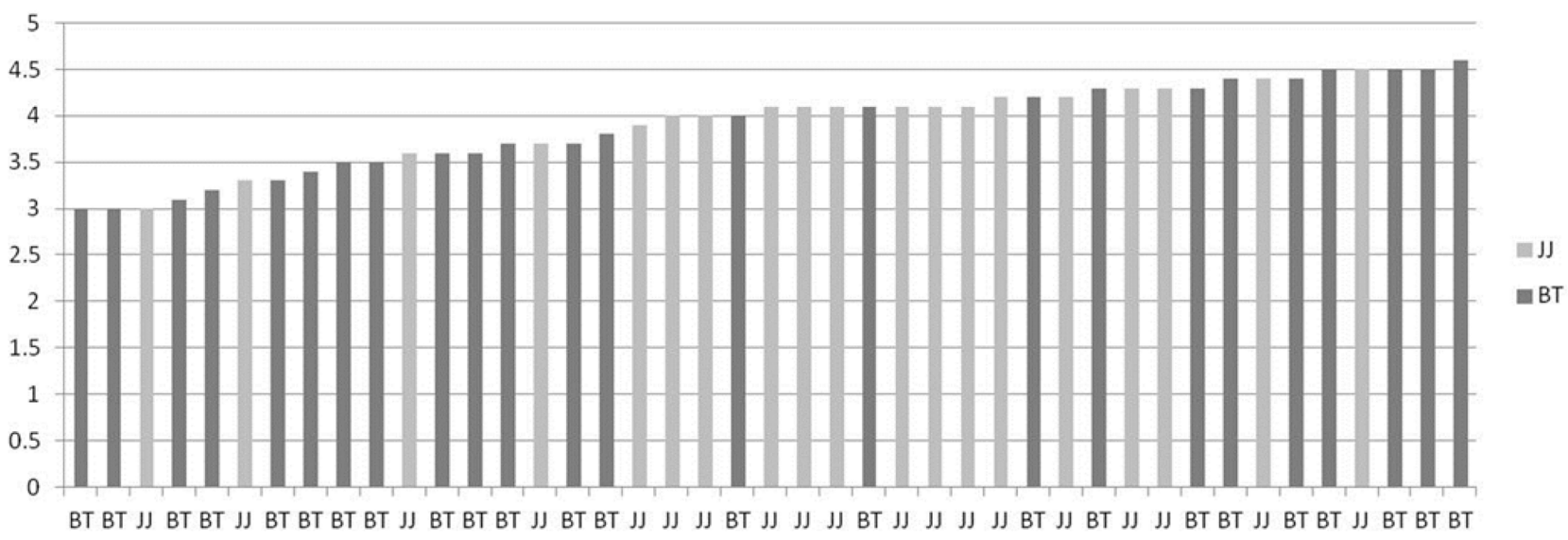

Fig. 4. Length of broad part of tube: Saussurea maximowiczii (BT) and S. maximowiczii var. triceps (JJ).

\section{Length of narrow part of tube}

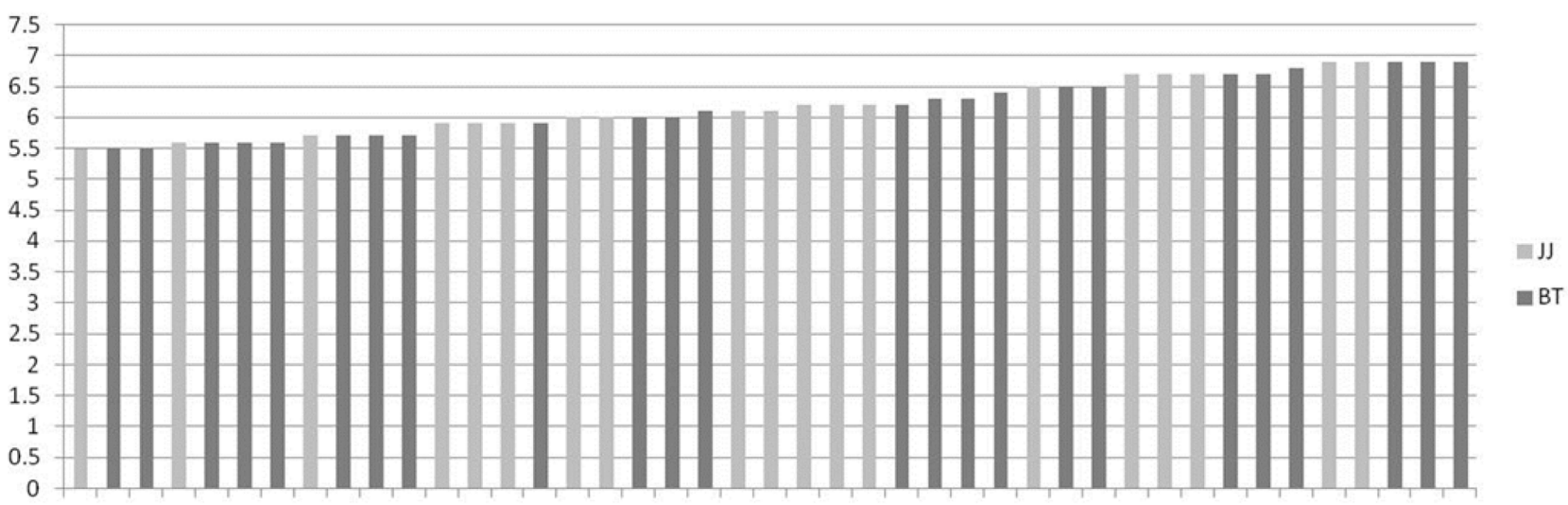

ر BT BT ر BT BT BT ر BT BT BT ر ر ر B B ر ر BT BT BT ر ر ر ر ر

Fig. 5. Length of broad part of tube: Saussurea maximowiczii (BT) and S. maximowiczii var. triceps (JJ). 


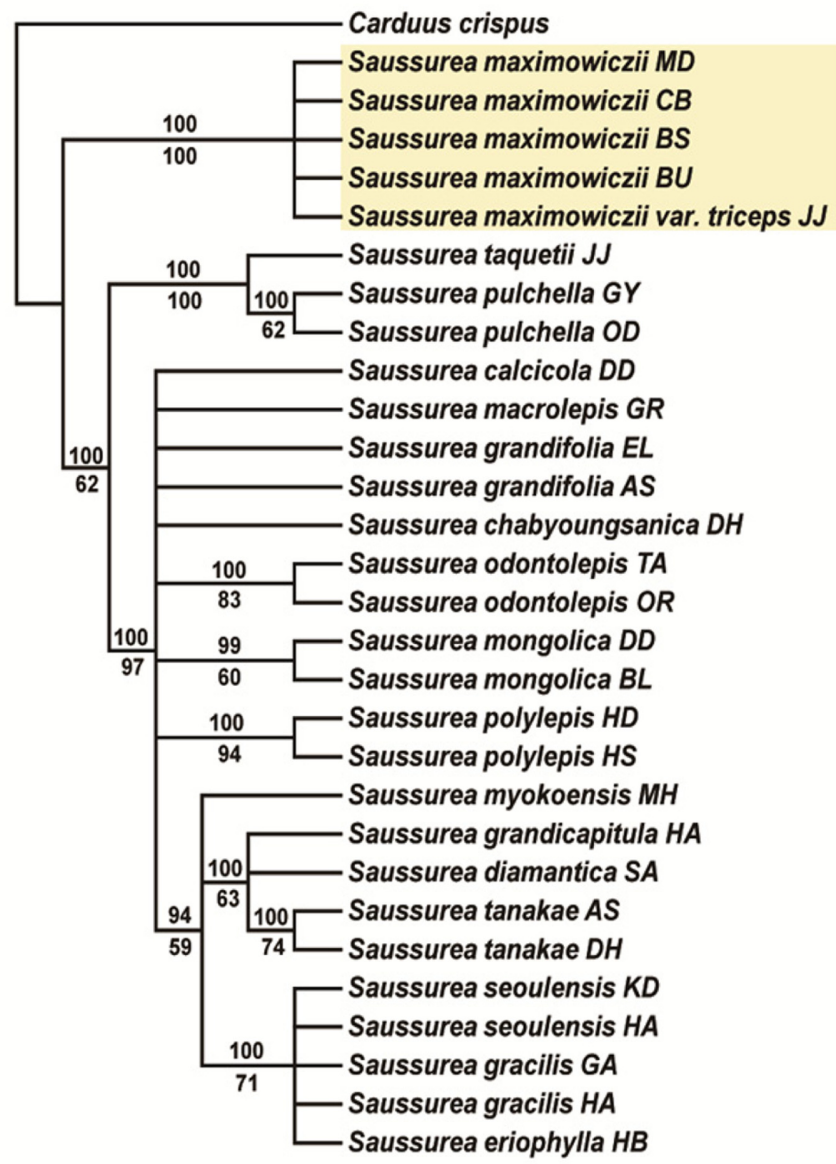

(B)

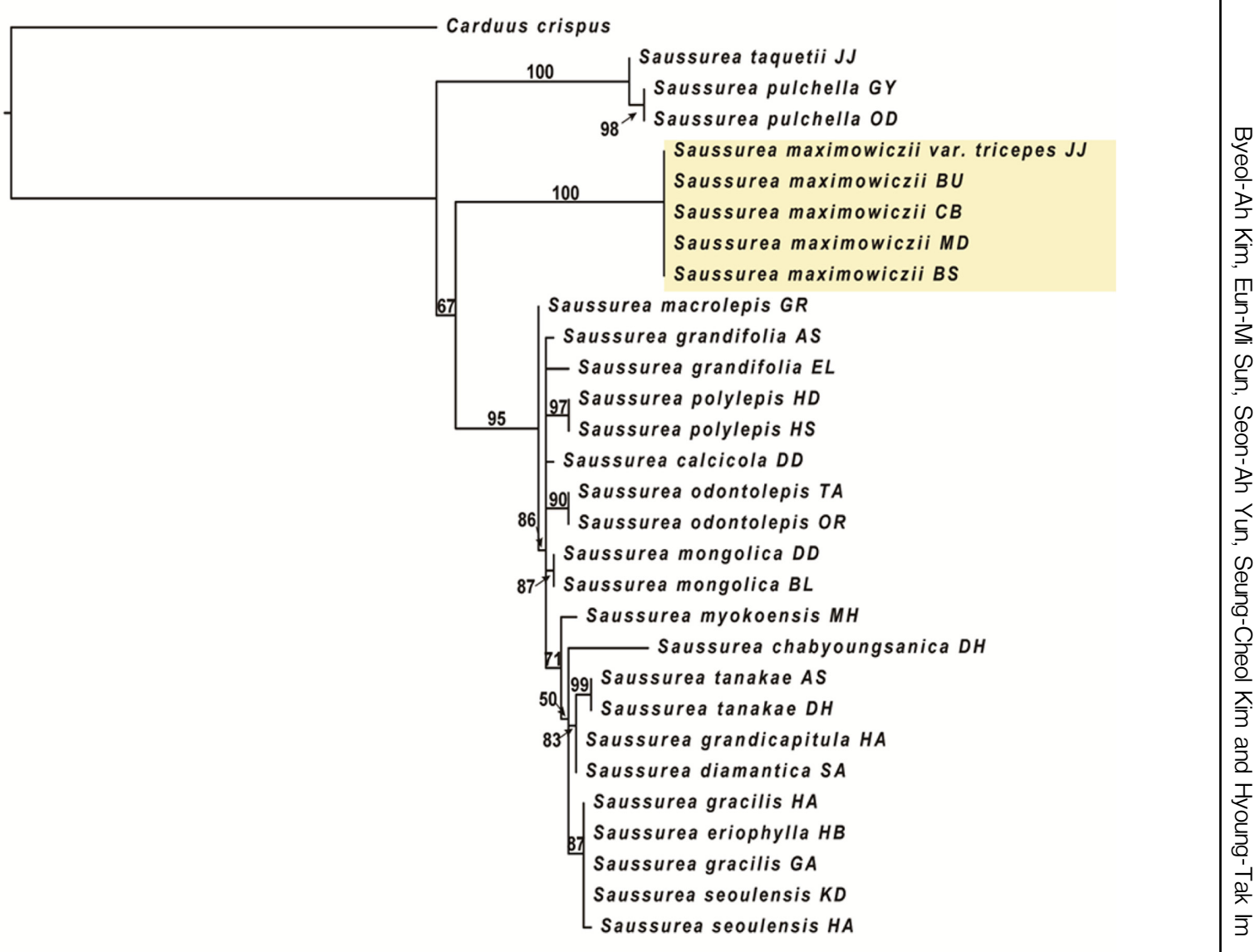

Fig. 6. Maximum parsimony (MP) tree and Maximum likelihood (ML) tree based on internal transcribed spacer sequences of 30 taxa including outgroup. A. Fifty percent majority consensus tree. Numbers below branches represent bootstrap support (BS) values (MP BS). B. ML tree. Numbers above branches represent bootstrap support values (ML BS) 
(A)

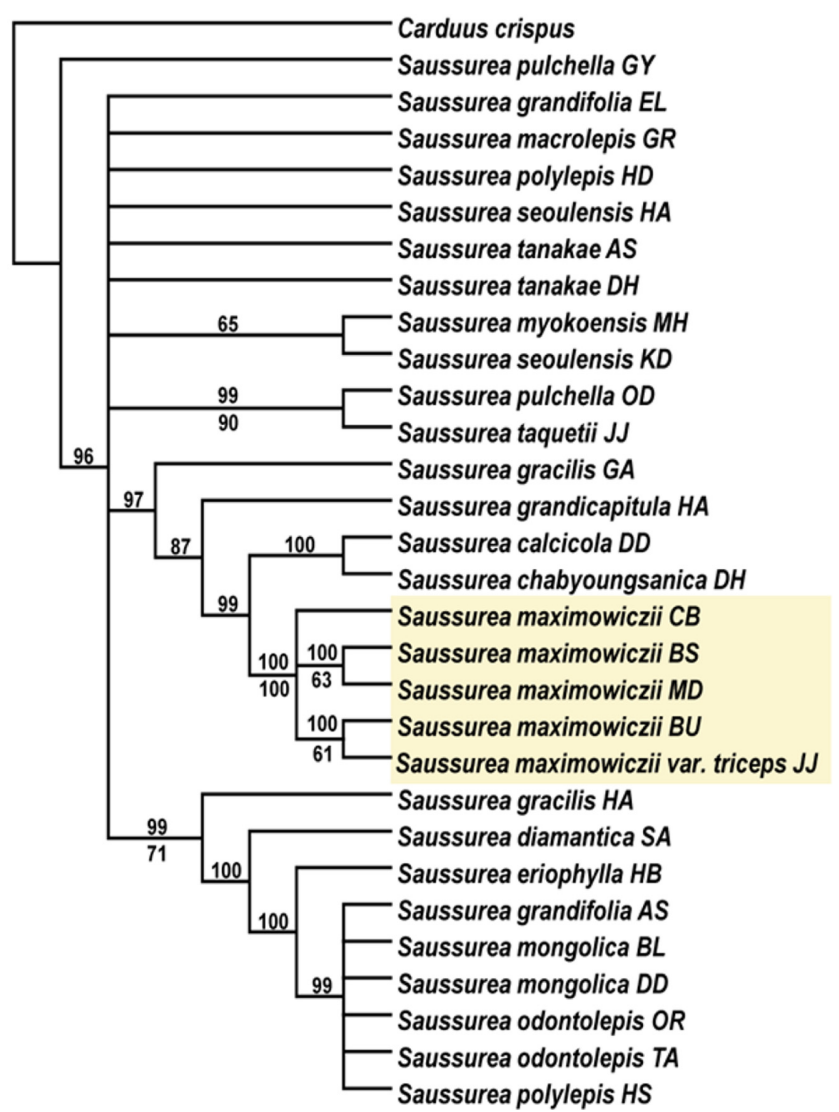

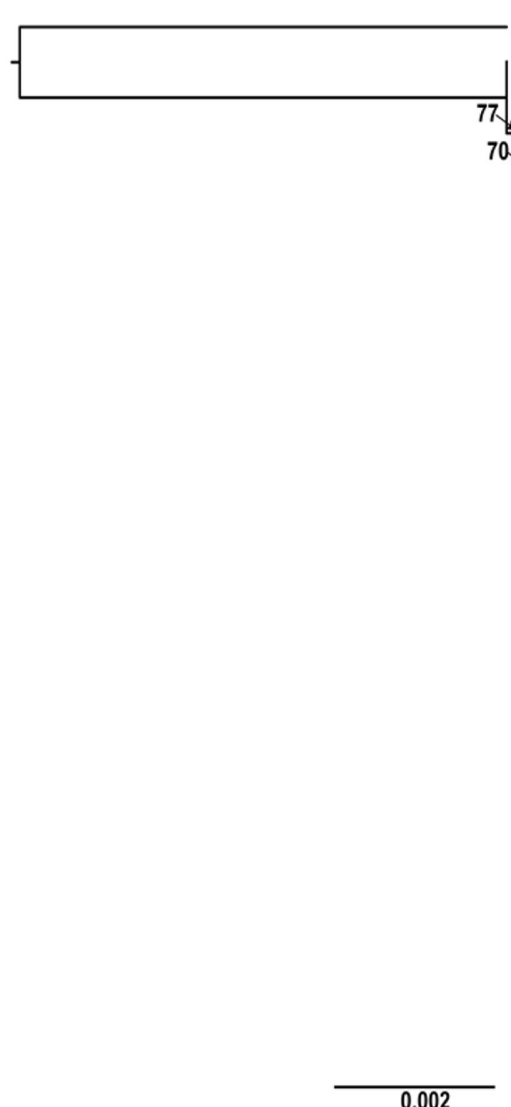

Carduus crispus

Saussurea seoulensis KD

Saussurea tanakae $D H$

- Saussurea polylepis HD

Saussurea seoulensis $H A$

Saussurea tanakae $A S$

Saussurea macrolepis GR

Saussurea grandifolia EL

64 Saussurea taquetii JJ

Saussurea myokoensis $M H$

Saussurea pulchella OD

Saussurea pulchella GY

- Saussurea grandicapitula $H A$

Saussurea gracilis GA

Saussurea maximowiczii $B S$

Saussurea maximowiczii MD

Saussurea maximowiczii BU

93 Saussurea maximowiczii var triceps JJ

- Saussurea gracilis $H A$

52 - Saussurea calcicola DD

Saussurea chabyoungsanica $D H$

Saussurea diamantica $S A$

Saussurea eriophylla $H B$

8 Saussurea polylepis HS

Saussurea grandifolia $A S$

Saussurea mongolica $D D$

Saussurea odontolepis $T A$

Saussurea mongolica $B L$

Saussurea odontolepis $O R$

Fig. 7. Maximum parsimony (MP) tree and Maximum likelihood (ML) tree based on combined cp sequences of 30 taxa including outgroup. A. Fifty percent majority consensus tree. Numbers below branches represent bootstrap support (BS) values (MP BS). B. ML tree. Numbers above branches represent bootstrap support values (ML BS). 
triceps is likely to continue to function as an independent species through peripheral isolation during local speciation process (Levin, 1993). To more clearly confirm the origin and taxonomic position of $S$. triceps, research results such as population genetics and others are needed.

\section{Taxonomic identity of Saussurea triceps - the ecophene}

In high elevation of Hallasan Mountain such as Yeongsil, Eorimok, and 1,100-m altitude wetlands, many plants are assumed to be ecophenes adapted to high mountain winds and low temperatures (Fig. 8). In the case of Aruncus aethusifolius (H. Lév.) Nakai, there is no difference between individuals of Yeongsil, the high mountain area (Fig. 8A), and individuals of Donnaeko above sea level of $400 \mathrm{~m}$. Both are about $20-30 \mathrm{~cm}$ tall. But in the case of Rubus oldhamii Miq., ordinary size of the terminal leaflet is $3-5 \times 2-3 \mathrm{~cm}$ in its main distribution region, forest margin of mountainous area. However, it is only about $1 \mathrm{~cm}$ in Yeongsil population (Fig. 8B). Ligularia fischeri
(Ledeb.) Turcz. of Eorimok dramatically shows change in shape between individuals on the windward side (Fig. 8C) and individuals on the downwind side (Fig. 8D). Individuals on the windward side are about $25 \mathrm{~cm}$ high with $6-8$ heads. On the other hand, individuals on the downwind (8D) only $10 \mathrm{~m}$ away from those of windward side $(8 \mathrm{C})$ are $60-70 \mathrm{~cm}$ high with $30-40$ heads.

Saussurea triceps also tends to be similar to Ligularia fischeri. Among specimens deposited at JNUB, there are individuals with stem lengths of $45 \mathrm{~cm}$ collected at the top of Hallasan Mountain (No. JJU0001401) (Table 1). Some specimens collected at $1,100-\mathrm{m}$ altitude wetland had stem lengths of $56 \mathrm{~cm}$ (No. JJU0013999) (Table 1). These individuals are presumed to have grown on the downwind side. Taking these morphological and molecular findings into considerations, S. triceps is an ecophene of S. maximowiczii that has adapted to the highland of Hallasan Mountain facing strong winds. Therefore, it is reasonable to recognize that $S$. triceps is a synonym of $S$. maximowiczii.
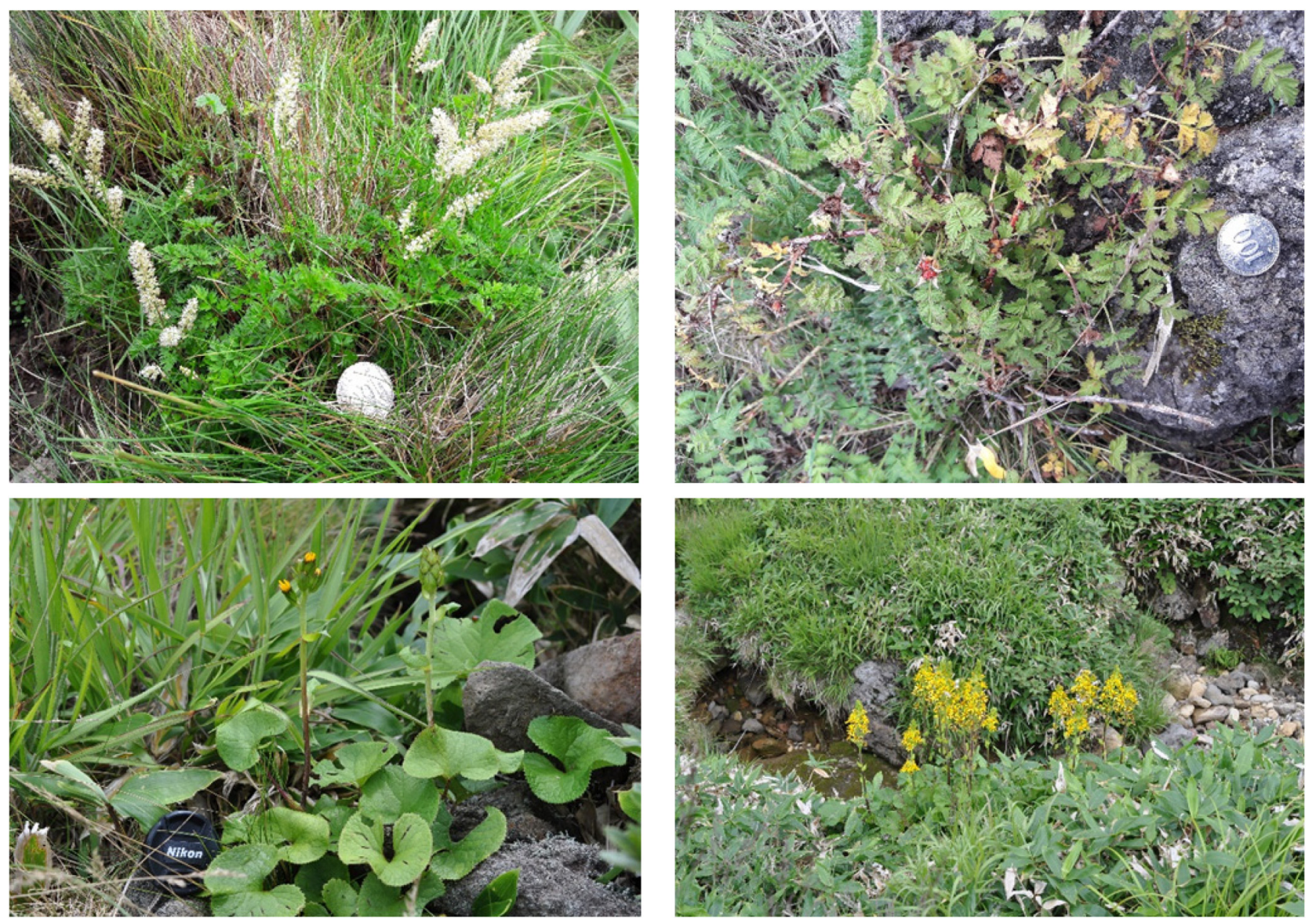

Fig. 8. Examples of ecophene adapted in highland of Hallasan Mountain, Jejudo Island. A. Aruncus aethusifolius (H. Lev.) Nakai. B. Rubus oldhamii Miq. C, D. Ligularia fischeri (Ledeb.) Turcz., C on windward side and D on downwind. 


\section{Taquet's taxa reported in Jejudo Island}

Among specimens collected by Taquet from Jejudo Island from 1907 to 1910, there are many new species described by H. Léveille and E. Vaniot, such as Allium taquetii $\mathrm{H}$. Lév. \& Vaniot (1908), Rosa taquetii Lev. (1909), Prunus taquetii H. Lév. (1909), Astilbe thunbergii var. taquetii $\mathrm{H}$. Lév. (1910), Astilbe thunbergii var. aethusifolius H. Lév. (1910), Senecio taquetii H. Lév. \& Vaniot (1910), Ophiopogon taquetii H. Lév. (1910), Chionanthus coreanus H. Lév. (1910), Matricaria coreana H. Lév. (1910), Salix blinii H. Lév. (1912), Salix hallaisanensis H. Lév. (1912), Ranunculus crucilobus H. Lév. (1912), Corydalis hallaisanensis H. Lév. (1912), Thalictrum punctatum $\mathrm{H}$. Lév. (1912), Pyrus brunnea H. Lév. (1912), Rosa quelpaertensis H. Lév. (1912), Cnicus rhincoceros H. Lév. \& Vaniot (1912), Cardamine glechomifolia H. Lév. (1913), Adenophora taquetii H. Lév. (1913), Aster hayatae H. Lév. \& Vaniot (1913), and so on. Some of these taxa underwent taxonomic treatment such as name combination or synonym while others are recognized as endemic species in Jejudo Island and Korea (NIBR, 2013).

Aruncus aethusifolius was originally reported to be a variety of Astilbe thunbergii (i.e., Astilbe thunbergii var. aethusifolia H. Lév., 1909). Later, it was changed to species Aruncus aethusifolius (H. Lév.) Nakai, 1912. Afterwards, it was downgraded to a variety (Aruncus dioicus var. aethusifolia (H. Lév.) Hara). Regardless its taxonomic position and rank (genus, species or infraspecific taxa) the taxonomic entity of Aruncus aethusifolius itself actually exists. In contrast, taxonomic identity of $S$. triceps is suspected since it has been treated as a synonym of other taxon frequently. To confirm or discuss taxonomic identity of taxa described based on nearly a century old collections such as A. aethusifolius or S. triceps, examination of field collected samples from type locality is necessary. Domestic researchers may play an important role in this regard because it is difficult for foreign researchers to access Korean endemic species.

\section{Conflict of Interest}

Authors declare that there is no conflict of interest.

\section{Acknowledgments}

This research was supported by the National Institute of Biological Resources as part of "Inventory and Management of Endemic Species of Korea” (NIBR 201706101).

\section{Literature Cited}

Chen, Y. and C. Shin. 1999. Compositae (8) Cyrareae. In Flora Republicae Popularis Sinicae, Tomus 78 (2). Chen, T. L. and C. Shih (eds.), Science Press, Peking. Pp. 100-102. (in Chinese)

Felsenstein, J. 1985. Confidence limits on phylogenies: an approach using the bootstrap. Evolution 39: 783-791.

Im, H. T. 1989. Taxonomic revision of the Saussurea nipponica complex (Compositae). Journal of the Faculty of Science, University of Tokyo, Section III 14: 243-272.

Im, H.-T. 1990. An instance of quantum speciation seen in Saussurea nipponica (Compositae). Korean Journal of Plant Taxonomy 20: 133-145.

Im, H.-T. 1991. Electrophoretic study of taxonomic relationships in the Saussurea nipponica complex (Compositae). Plant Species Biology 6: 11-18.

Im, R. J. and E. C. Lee. 1999. Compositae. In Flora Coreana. Vol. 7. Im, R. J. (ed.), Science and Technology Publishing House, Pyongyang. Pp. 70-116.

Kearse, M., R. Moir, A. Wilson, S. Stones-Havas, M. Cheung, S. Sturrock, S. Buxton, A. Cooper, S. Markowitz, C. Duran, T. Thierer, B. Ashton, P. Meintjes and A. Drummond. 2012. Geneious Basic: an integrated and extendable desktop software platform for the organization and analysis of sequence data. Bioinformatics 28: 1647-1649.

Kita, Y., K. Fujikawa, M. Ito, H. Ohba and M. Kato. 2004. Molecular phylogenetic analyses and systematics of the genus Saussurea and related genera (Asteraceae, Cardueae). Taxon 53: 679-690.

Kitamura, S. 1937. Compositae Japonicae. Memoirs of the College of Science, Kyoto Imperial University, Series B Biology 13: $1-421$.

Lee, W. T. 1996. Linneamenta Florae Koreae. Academy Publishing Co., Seoul. Pp. 1179-1191.

Levin, D. A. 1993. Local speciation in plants: the rule not the exception. Systematic Botany 18: 197-208.

Lipschitz, S. 1979. Genus Saussurea DC. (Asteraceae). Nauka, Leningrad, $281 \mathrm{pp}$.

Nguyen, L. T., H. A. Schmidt, A. von Haeseler and B. Q. Minh. 2015. IQ-TREE: a fast and effective stochastic algorithm for estimating maximum-likelihood phylogenies. Molecular Biology and Evolution 32: 268-274.

NIBR. 2013. Endemic Species of Korea. National Institute of Biological Resources. Duhyeon Publishing, Seoul, 912 pp.

Shaw, J., E. B. Lickey, E. E. Schilling and R. L. Small. 2007. Comparison of whole chloroplast genome sequences to choose noncoding regions for phylogenetic studies in angio- 
sperms: the tortoise and the hare III. American Journal of Botany 94: 275-288.

Swofford, D. L. 2001. PAUP*: Phylogenetic Analysis Using Parsimony (*and other methods) version 4.0. Sinauer Associates, Sunderland, MA.

Von Raab-Straube, E. 2003. Phylogenetic relationships in Saussurea (Compositae, Cardueae) sensu lato, inferred from morphological, ITS and trnL-trnF sequence data, with a synopsis of Himalaiella gen. nov., Lipschitziella and Frolovia. Willdenowia 33: 379-402.
Wang, Y.-J., J.-Q. Liu and G. Miehe. 2007. Phylogenetic origins of the Himalayan endemic Dolomiaea, Diplazoptilon and Xanthopappus (Asteraceae: Cardueae) based on three DNA regions. Annals of Botany 99: 311-322.

Wang, Y.-J., A. Susanna, E. von Raab-Straube, R. Milne. and J.-Q. Liu. 2009. Island-like radiation of Saussurea (Asteraceae: Cardueae) triggered by uplifts of the Qinghai-Tibetan Plateau. Biological Journal of the Linnean Society 97: 893-903.

Wang, Y.-J., E. von Raab-Straube, A. Susanna and J.-Q. Liu. 2013. Shangwua (Compositae), a new genus from the QinghaiTibetan Plateau and Himalayas. Taxon 62: 984-996.

\title{
한라분취의 분류학적 인식
}

\author{
김별아 · 선은미 · 윤선아 ${ }^{1} \cdot$ 김승철 ${ }^{1}$ - 임형탁* \\ 전남대학교 자연과학대학 생물학과, ${ }^{1}$ 성균관대학교 생명과학과
}

적 요: 한라분취(Saussurea maximowiczii var. triceps)의 분류학적 실체에 대해 형태 및 분자적 접근을 했다. 줄기 길이, 잎의 크기와 같은 영양기관의 정량형질에서 한라분취가 버들분취(S. maximowiczii Herd.)보다 작 은 경향이 있으나, 이는 연속적 변이이다. 소화, 총포를 비롯한 생식기관 성질에서 한라분취와 버들분취는 구별할 수 없었다. 버들분취와 한라분취의 염기서열을 확인한 결과 이들은 단계통을 이루었다. 한라분취는 강풍에 면한 한라산 고산지대에 적응한 버들분취의 생태표현형으로 추정된다.

주요어: 한라분취, 생태형, 고지, 제주도 